ANNALES

POLONICI MATHEMATICI

$89.3(2006)$

\title{
On uniqueness for nonlinear differential polynomials sharing the same 1-point
}

\author{
by AbHiJit BanerJee (Kalyani)
}

\begin{abstract}
We study the uniqueness of meromorphic functions when two nonlinear differential polynomials generated by two meromorphic functions share the same 1-points. Our results improve results of Fang-Fang and Lin-Yi and supplement a recent result of Lahiri-Pal.
\end{abstract}

1. Introduction, definitions and results. Let $f$ and $g$ be two nonconstant meromorphic functions defined in the open complex plane $\mathbb{C}$. If for some $a \in \mathbb{C} \cup\{\infty\}, f-a$ and $g-a$ have the same set of zeros with the same multiplicities, we say that $f$ and $g$ share the value a $C M$ (counting multiplicities), and if we do not consider the multiplicities then $f$ and $g$ are said to share the value a IM (ignoring multiplicities).

Let $m$ be a positive integer or infinity and $a \in \mathbb{C} \cup\{\infty\}$. We denote by $E_{m)}(a ; f)$ the set of all $a$-points of $f$ with multiplicities not exceeding $m$, where an $a$-point is counted according to its multiplicity. Also we denote by $\bar{E}_{m)}(a ; f)$ the set of distinct $a$-points of $f(z)$ with multiplicities not greater than $m$. If for some $a \in \mathbb{C} \cup\{\infty\}, E_{\infty)}(a ; f)=E_{\infty)}(a ; g)$ we say that $f, g$ share the value a $C M$.

We denote by $T(r)$ the maximum of $T(r, f)$ and $T(r, g)$. The notation $S(r)$ indicates any quantity satisfying $S(r)=o(T(r))$ as $r \rightarrow \infty$, outside of a possible exceptional set of finite linear measure.

We use $I$ to denote any set of $0<r<\infty$ of infinite linear measure.

During the last few years a great deal of research has been carried out on the uniqueness of meromorphic functions generating differential polynomials (cf. [1], [2], [3], [5], [9], [12]-[15]). In [5] and [9] Lahiri studied the problem of uniqueness of meromorphic functions when two linear differential polynomials generated by them share the same 1-points.

2000 Mathematics Subject Classification: Primary 30D35.

Key words and phrases: uniqueness, meromorphic function, nonlinear differential polynomial, shared value. 
In [5] the following question was asked.

What can be said if two nonlinear differential polynomials generated by two meromorphic functions share 1 CM?

A considerable amount of research has already been done in this direction. (cf. [1]-[3], [12]-[15]). In 2002 Fang and Fang [2] and in 2004 Lin-Yi [15] independently proved the following result.

THEOREM A. Let $f$ and $g$ be two nonconstant meromorphic functions and $n \geq 13$ be an integer. If $f^{n}(f-1)^{2} f^{\prime}$ and $g^{n}(g-1)^{2} g^{\prime}$ share $1 C M$, then $f \equiv g$.

Also in [2] Fang and Fang proved the following theorem.

THEOREM B. Let $f$ and $g$ be two nonconstant meromorphic functions and $n \geq 28$ be an integer. If $f^{n}(f-1)^{2} f^{\prime}$ and $g^{n}(g-1)^{2} g^{\prime}$ share 1 IM, then $f \equiv g$.

In [2] Fang and Fang and recently in [12] Lahiri-Pal proved the following result.

TheOREM C. Let $f$ and $g$ be two nonconstant meromorphic functions and $n \geq 13$ be an integer. If $E_{3)}\left(1 ; f^{n}(f-1)^{2} f^{\prime}\right)=E_{3)}\left(1 ; g^{n}(g-1)^{2} g^{\prime}\right)$, then $f \equiv g$.

In 2001 an idea of gradation of sharing of values was introduced in [7], [8] to measure how close a shared value is to being shared CM or IM. This notion is known as weighted sharing and is defined as follows.

Definition 1.1 ([7], [8]). Let $k$ be a nonnegative integer or infinity. For $a \in \mathbb{C} \cup\{\infty\}$ we denote by $E_{k}(a ; f)$ the set of all $a$-points of $f$, where an $a$-point of multiplicity $m$ is counted $m$ times if $m \leq k$ and $k+1$ times if $m>k$. If $E_{k}(a ; f)=E_{k}(a ; g)$, we say that $f, g$ share the value a with weight $k$.

The definition implies that if $f, g$ share a value $a$ with weight $k$ then $z_{0}$ is an $a$-point of $f$ with multiplicity $m \leq k$ if and only if it is an $a$-point of $g$ with multiplicity $m \leq k$, and $z_{0}$ is an $a$-point of $f$ with multiplicity $m>k$ if and only if it is an $a$-point of $g$ with multiplicity $n>k$, where $m$ is not necessarily equal to $n$.

We write $f, g$ share $(a, k)$ to mean that $f, g$ share the value $a$ with weight $k$. Clearly if $f, g$ share $(a, k)$, then $f, g$ share $(a, p)$ for any integer $p$, $0 \leq p<k$. Also we note that $f, g$ share a value $a$ IM or CM if and only if $f, g$ share $(a, 0)$ or $(a, \infty)$ respectively.

In this paper we employ the idea of weighted sharing of values and truncated sharing of values to investigate the uniqueness of meromorphic functions when the nonlinear differential polynomials of the form mentioned in 
Theorem A share the value 1. We now give the following five theorems which are our main results.

THEOREM 1.1. Let $f$ and $g$ be two nonconstant meromorphic functions and $n>12-2 \Theta(\infty ; f)-2 \Theta(\infty ; g)-\min \{\Theta(\infty ; f), \Theta(\infty ; g)\}$ be an integer. If $f^{n}(f-1)^{2} f^{\prime}$ and $g^{n}(g-1)^{2} g^{\prime}$ share $(1,2)$ then $f \equiv g$.

Remark 1.1. If in Theorem 1.1 we take $\min \{\Theta(\infty ; f), \Theta(\infty ; g)\}>0$ then the assertion is true for $n \geq 12$. Clearly Theorem 1.1 is an improvement of Theorem A.

THEOREM 1.2. Let $f$ and $g$ be two nonconstant meromorphic functions and $n>29 / 2-3 \Theta(\infty ; f)-3 \Theta(\infty ; g)$ be an integer. If $f^{n}(f-1)^{2} f^{\prime}$ and $g^{n}(g-1)^{2} g^{\prime}$ share $(1,1)$ then $f \equiv g$.

THEOREM 1.3. Let $f$ and $g$ be two nonconstant meromorphic functions and $n>27-5 \Theta(\infty ; f)-5 \Theta(\infty ; g)-\min \{\Theta(\infty ; f), \Theta(\infty ; g)\}$ be an integer. If $f^{n}(f-1)^{2} f^{\prime}$ and $g^{n}(g-1)^{2} g^{\prime}$ share $(1,0)$ then $f \equiv g$.

Remark 1.2. If in Theorem 1.3 we take $\min \{\Theta(\infty ; f), \Theta(\infty ; g)\}>0$ then the assertion is true for $n \geq 27$.

THEOREM 1.4. Let $f$ and $g$ be two nonconstant meromorphic functions and $n \geq 12$ be an integer. If

$$
\begin{aligned}
& \bar{E}_{4)}\left(1 ; f^{n}(f-1)^{2} f^{\prime}\right)=\bar{E}_{4)}\left(1 ; g^{n}(g-1)^{2} g^{\prime}\right), \\
& E_{2)}\left(1 ; f^{n}(f-1)^{2} f^{\prime}\right)=E_{2)}\left(1 ; g^{n}(g-1)^{2} g^{\prime}\right)
\end{aligned}
$$

and $\min \{\Theta(\infty ; f), \Theta(\infty ; g)\}>0$ then $f \equiv g$.

REMARK 1.3. If in Theorem 1.4 we take $n \geq 13$ then the condition $\min \{\Theta(\infty ; f), \Theta(\infty ; g)\}>0$ can be removed and in that case Theorem 1.4 improves Theorem A.

THEOREM 1.5. Let $f$ and $g$ be two nonconstant meromorphic functions and $n \geq 13$ be an integer. If

$$
\begin{aligned}
& \bar{E}_{3)}\left(1 ; f^{n}(f-1)^{2} f^{\prime}\right)=\bar{E}_{3)}\left(1 ; g^{n}(g-1)^{2} g^{\prime}\right), \\
& E_{1)}\left(1 ; f^{n}(f-1)^{2} f^{\prime}\right)=E_{1)}\left(1 ; g^{n}(g-1)^{2} g^{\prime}\right)
\end{aligned}
$$

and $\min \{\Theta(\infty ; f), \Theta(\infty ; g)\}>1 / 4$ then $f \equiv g$.

REMARK 1.4. If in Theorem 1.5 we take $n \geq 15$ then the condition $\min \{\Theta(\infty ; f), \Theta(\infty ; g)\}>1 / 4$ can be removed.

Though we use the standard notations and definitions of the value distribution theory available in [4], we explain some definitions and notations which are used in the paper.

Definition $1.2([6])$. For $a \in \mathbb{C} \cup\{\infty\}$ we denote by $N(r, a ; f \mid=1)$ the counting function of simple $a$-points of $f$. For a positive integer $m$ we 
denote by $N(r, a ; f \mid \leq m)$ (resp. $N(r, a ; f \mid \geq m)$ ) the counting function of those $a$-points of $f$ whose multiplicities are not greater (resp. less) than $m$ where each $a$-point is counted according to its multiplicity.

$\bar{N}(r, a ; f \mid \leq m)$ and $\bar{N}(r, a ; f \mid \geq m)$ are defined similarly, but ignoring multiplicities.

Also $N(r, a ; f \mid<m), N(r, a ; f \mid>m), \bar{N}(r, a ; f \mid<m)$ and $\bar{N}(r, a ; f \mid>m)$ are defined analogously.

Definition 1.3 ([8], cf. [20]). We denote by $N_{2}(r, a ; f)$ the sum $\bar{N}(r, a ; f)+\bar{N}(r, a ; f \mid \geq 2)$.

DeFinition 1.4. Let $m$ and $r$ be two positive integers such that $1 \leq r<$ $m-1$ and for some $a \in \mathbb{C}, \bar{E}_{m)}(a ; f)=\bar{E}_{m)}(a ; g), E_{r)}(a ; f)=E_{r)}(a ; g)$. Let $z_{0}$ be a zero of $f(z)-a$ of multiplicity $p$ and a zero of $g(z)-a$ of multiplicity $q$. We denote by $\bar{N}_{L}(r, a ; f)$ (resp. $\left.\bar{N}_{L}(r, a ; g)\right)$ the reduced counting function of those $a$-points of $f$ and $g$ for which $p>q \geq r+1$ (resp. $q>p \geq$ $r+1)$, by $\bar{N}_{E}^{(r+1}(r, a ; f)$ the reduced counting function of those $a$-points of $f$ and $g$ for which $p=q \geq r+1$, and by $\bar{N}_{f \geq m+1}(r, a ; f \mid g \neq a)$ (resp. $\left.\bar{N}_{g \geq m+1}(r, a ; g \mid f \neq a)\right)$ the reduced counting functions of those $a$-points of $f$ and $g$ for which $p \geq m+1$ and $q=0$ (resp. $q \geq m+1$ and $p=0$ ).

Definition 1.5. Let $\bar{E}_{m)}(a ; f)=\bar{E}_{m)}(a ; g)$ for some $a \in \mathbb{C}$. Also let $z_{0}$ be a zero of $f-a$ of multiplicity $p$ and a zero of $g-a$ of multiplicity $q$. We denote by $\bar{N}(r, a ; f|=p ; g|=q)$ the reduced counting functions of common $a$-points of $f$ and $g$ with multiplicity $p$ and $q$ respectively. Also we denote by $\bar{N}_{f>s}(r, a ; g)\left(\operatorname{resp} . \bar{N}_{f>s}(r, a ; g)\right)$ the reduced counting functions of those $a$-points of $f$ and $g$ for which $p>q=s$ (resp. $q>p=s$ ).

Definition $1.6([10])$. Let $a, b \in \mathbb{C} \cup\{\infty\}$. We denote by $N(r, a ; f \mid g=b)$ the counting function of those $a$-points of $f$, counted according to multiplicity, which are $b$-points of $g$.

Definition $1.7([10])$. Let $a, b \in \mathbb{C} \cup\{\infty\}$. We denote by $N(r, a ; f \mid g \neq b)$ the counting function of those $a$-points of $f$, counted according to multiplicity, which are not $b$-points of $g$.

2. Lemmas. In this section we present some lemmas which will be needed in what follows. Let $f, g, F, G$ be four nonconstant meromorphic functions. Henceforth we shall denote by $h$ and $H$ the following two functions:

$$
\begin{aligned}
h & =\left(\frac{f^{\prime \prime}}{f^{\prime}}-\frac{2 f^{\prime}}{f-1}\right)-\left(\frac{g^{\prime \prime}}{g^{\prime}}-\frac{2 g^{\prime}}{g-1}\right), \\
H & =\left(\frac{F^{\prime \prime \prime}}{F^{\prime \prime}}-\frac{2 F^{\prime \prime}}{F^{\prime}-1}\right)-\left(\frac{G^{\prime \prime \prime}}{G^{\prime \prime}}-\frac{2 G^{\prime \prime}}{G^{\prime}-1}\right) .
\end{aligned}
$$


LEMMA 2.1. If $f, g$ are two nonconstant meromorphic functions such that $E_{1)}(1 ; f)=E_{1)}(1 ; g)$ and $h \not \equiv 0$ then

$N(r, 1 ; f \mid=1)=N(r, 1 ; g \mid=1) \leq N(r, 0 ; h) \leq N(r, \infty ; h)+S(r, f)+S(r, g)$.

Proof. Since $f$ and $g$ have the same simple 1-points it can be easily verified by direct computation that $h$ is zero whenever $f-1$ has a simple zero. This proves the lemma.

Lemma 2.2. Let $\bar{E}_{m)}(1 ; f)=\bar{E}_{m)}(1 ; g), E_{1)}(1 ; f)=E_{1)}(1 ; g)$ and $h \not \equiv 0$, where $m \geq 3$. Then

$$
\begin{aligned}
N(r, \infty ; h) \leq & \bar{N}(r, 0 ; f \mid \geq 2)+\bar{N}(r, 0 ; g \mid \geq 2)+\bar{N}(r, \infty ; f \mid \geq 2) \\
& +\bar{N}(r, \infty ; g \mid \geq 2)+\bar{N}_{L}(r, 1 ; f)+\bar{N}_{L}(r, 1 ; g) \\
& +\bar{N}_{f \geq m+1}(r, 1 ; f \mid g \neq 1)+\bar{N}_{g \geq m+1}(r, 1 ; g \mid f \neq 1) \\
& +\bar{N}_{0}\left(r, 0 ; f^{\prime}\right)+\bar{N}_{0}\left(r, 0 ; g^{\prime}\right),
\end{aligned}
$$

where $\bar{N}_{0}\left(r, 0 ; f^{\prime}\right)$ is the reduced counting function of those zeros of $f^{\prime}$ which are not the zeros of $f(f-1)$, and $\bar{N}_{0}\left(r, 0 ; g^{\prime}\right)$ is similarly defined.

Proof. We can easily verify that possible poles of $h$ occur at (i) multiple zeros of $f$ and $g$, (ii) multiple poles of $f$ and $g$, (iii) common zeros of $f-1$ and $g-1$ whose multiplicities are different, (iv) those 1-points of $f$ (or $g$ ) which are not 1-points of $g$ (or $f$ ), (v) zeros of $f^{\prime}$ which are not zeros of $f(f-1)$, (vi) zeros of $g^{\prime}$ which are not zeros of $g(g-1)$.

Since all poles of $h$ are simple, the lemma follows from the above.

LEMMA $2.3([11])$. If $N\left(r, 0 ; f^{(k)} \mid f \neq 0\right)$ denotes the counting function of those zeros of $f^{(k)}$ which are not zeros of $f$, where a zero of $f^{(k)}$ is counted according to its multiplicity, then

$$
\begin{aligned}
N\left(r, 0 ; f^{(k)} \mid f \neq 0\right) \leq & k \bar{N}(r, \infty ; f)+N(r, 0 ; f \mid<k) \\
& +k \bar{N}(r, 0 ; f \mid \geq k)+S(r, f) .
\end{aligned}
$$

LEMMA 2.4 (cf. [16], [17]). Let $f$ be a nonconstant meromorphic function and $P(f)=a_{0}+a_{1} f+\cdots+a_{n} f^{n}$, where $a_{0}, a_{1}, \ldots, a_{n}$ are constants and $a_{n} \neq 0$. Then $T(r, P(f))=n T(r, f)+O(1)$.

LEMMA 2.5. If $\bar{E}_{3)}(1 ; f)=\bar{E}_{3)}(1 ; g)$ and $E_{1)}(1 ; f)=E_{1)}(1 ; g)$, then

$$
\begin{aligned}
2 \bar{N}_{L}(r, 1 ; f)+2 \bar{N}_{L}(r, 1 ; g)+ & \bar{N}_{E}^{(2}(r, 1 ; f) \\
& +3 \bar{N}_{g \geq 4}(r, 1 ; g \mid f \neq 1)-\bar{N}_{f>2}(r, 1 ; g) \\
\leq & N(r, 1 ; g)-\bar{N}(r, 1 ; g) .
\end{aligned}
$$

Proof. Let $z_{0}$ be a 1-point of $f$ with multiplicity $p$ and a 1-point of $g$ with multiplicity $q$. If $q=2$ the possible values of $p$ are (i) $p=2$, (ii) $p=3$. When $q=3$, the possible values of $p$ are (i) $p=2$ (ii), $p=3$. If $q=4$ the possible values of $p$ are (i) $p=4$, (ii) $p \geq 5$, (iii) $p=0$. Similarly when $q=5$ the possible values of $p$ are (i) $p=4$, (ii) $p=5$, (iii) $p \geq 6$, (iv) $p=0$. If $q \geq 6$ 
we can similarly find the possible values of $p$. The lemma now follows from the above discussion since the simple 1-points of $f$ and $g$ are the same.

Lemma 2.6. If $\bar{E}_{3)}(1 ; f)=\bar{E}_{3)}(1 ; g)$ and $E_{1)}(1 ; f)=E_{1)}(1 ; g)$ then

(ii) $\quad \bar{N}_{g>2}(r, 1 ; f) \leq \frac{1}{2} \bar{N}(r, 0 ; g)+\frac{1}{2} \bar{N}(r, \infty ; g)-\frac{1}{2} N_{0}\left(r, 0 ; g^{\prime}\right)+S(r, g)$.

Proof. We prove (i) since the proof of (ii) can be carried out similarly.

Using Lemma 2.3 we get

$$
\begin{aligned}
\bar{N}_{f>2}(r, 1 ; g) & \leq \bar{N}(r, 1 ; f \mid \geq 3) \leq \frac{1}{2} N\left(r, 0 ; f^{\prime} \mid f=1\right) \\
& \leq \frac{1}{2} \bar{N}(r, 0 ; f)+\frac{1}{2} \bar{N}(r, \infty ; f)-\frac{1}{2} N_{0}\left(r, 0 ; f^{\prime}\right)+S(r, f) .
\end{aligned}
$$

Lemma 2.7. Let $\bar{E}_{3)}(1 ; f)=\bar{E}_{3)}(1 ; g), E_{1)}(1 ; f)=E_{1)}(1 ; g)$ and $h \not \equiv 0$. Then

$$
\begin{aligned}
T(r, f)+T(r, g) \leq & 2\left\{N_{2}(r, 0 ; f)+N_{2}(r, \infty ; f)+N_{2}(r, 0 ; g)+N_{2}(r, \infty ; g)\right\} \\
& +\frac{1}{2}[\bar{N}(r, 0 ; f)+\bar{N}(r, \infty ; f)+\bar{N}(r, 0 ; g)+\bar{N}(r, \infty ; g)] \\
& -m(r, 1 ; f)-m(r, 1 ; g)+S(r, f)+S(r, g) .
\end{aligned}
$$

Proof. By the second fundamental theorem we get

$$
\begin{aligned}
T(r, f)+T(r, g) \leq & \bar{N}(r, 0 ; f)+\bar{N}(r, \infty ; f)+\bar{N}(r, 0 ; g)+\bar{N}(r, \infty ; g) \\
& +\bar{N}(r, 1 ; f)+\bar{N}(r, 1 ; g)-N_{0}\left(r, 0 ; f^{\prime}\right) \\
& -N_{0}\left(r, 0 ; g^{\prime}\right)+S(r, f)+S(r, g) .
\end{aligned}
$$

By Lemmas 2.1, 2.2 and 2.5 we get

$$
\begin{aligned}
\bar{N}(r, 1 ; f)+ & \bar{N}(r, 1 ; g) \\
\leq & N(r, 1 ; f \mid=1)+\bar{N}_{L}(r, 1 ; f)+\bar{N}_{L}(r, 1 ; g) \\
& +\bar{N}_{E}^{(2}(r, 1 ; f)+\bar{N}_{f \geq 4}(r, 1 ; f \mid g \neq 1)+\bar{N}(r, 1 ; g) \\
\leq & \bar{N}(r, 0 ; f \mid \geq 2)+\bar{N}(r, \infty ; f \mid \geq 2)+\bar{N}(r, 0 ; g \mid \geq 2) \\
& +\bar{N}(r, \infty ; g \mid \geq 2)+\bar{N}_{f \geq 4}(r, 1 ; f \mid g \neq 1) \\
& +\bar{N}_{g \geq 4}(r, 1 ; g \mid f \neq 1)+\bar{N}_{L}(r, 1 ; f)+\bar{N}_{L}(r, 1 ; g) \\
& +\bar{N}_{0}\left(r, 0 ; f^{\prime}\right)+\bar{N}_{0}\left(r, 0 ; g^{\prime}\right)+\bar{N}_{L}(r, 1 ; f) \\
& +\bar{N}_{L}(r, 1 ; g)+\bar{N}_{E}^{(2}(r, 1 ; f)+\bar{N}_{f \geq 4}(r, 1 ; f \mid g \neq 1) \\
& +N(r, 1 ; g)-2 \bar{N}_{L}(r, 1 ; f)-2 \bar{N}_{L}(r, 1 ; g) \\
& -\bar{N}_{E}^{(2}(r, 1 ; f)-3 \bar{N}_{g \geq 4}(r, 1 ; g \mid f \neq 1) \\
& +\bar{N}_{f>2}(r, 1 ; g)+S(r, f)+S(r, g)
\end{aligned}
$$




$$
\begin{aligned}
\leq & \bar{N}(r, 0 ; f \mid \geq 2)+\bar{N}(r, \infty ; f \mid \geq 2)+\bar{N}(r, 0 ; g \mid \geq 2) \\
& +\bar{N}(r, \infty ; g \mid \geq 2)+2 \bar{N}_{f \geq 4}(r, 1 ; f \mid g \neq 1) \\
& -2 \bar{N}_{g \geq 4}(r, 1 ; g \mid f \neq 1)+\bar{N}_{f>2}(r, 1 ; g)+T(r, g)-m(r, 1 ; g) \\
& +\bar{N}_{0}\left(r, 0 ; f^{\prime}\right)+\bar{N}_{0}\left(r, 0 ; g^{\prime}\right)+S(r, f)+S(r, g) .
\end{aligned}
$$

Using Lemma 2.6 we deduce from (2.1) and (2.2) that

$$
\begin{aligned}
T(r, f) \leq & N_{2}(r, 0 ; f)+N_{2}(r, \infty ; f)+N_{2}(r, 0 ; g)+N_{2}(r, \infty ; g) \\
& +2 \bar{N}_{f \geq 4}(r, 1 ; f \mid g \neq 1)-2 \bar{N}_{g \geq 4}(r, 1 ; g \mid f \neq 1) \\
& +\frac{1}{2} \bar{N}(r, 0 ; f)+\frac{1}{2} \bar{N}(r, \infty ; f)-m(r, 1 ; g) \\
& +S(r, f)+S(r, g) .
\end{aligned}
$$

Similarly we can obtain

$$
\begin{aligned}
T(r, g) \leq & N_{2}(r, 0 ; f)+N_{2}(r, \infty ; f)+N_{2}(r, 0 ; g)+N_{2}(r, \infty ; g) \\
& +2 \bar{N}_{g \geq 4}(r, 1 ; g \mid f \neq 1)-2 \bar{N}_{f \geq 4}(r, 1 ; f \mid g \neq 1) \\
& +\frac{1}{2} \bar{N}(r, 0 ; g)+\frac{1}{2} \bar{N}(r, \infty ; g)-m(r, 1 ; f) \\
& +S(r, f)+S(r, g) .
\end{aligned}
$$

Adding (2.3) and (2.4) we get the conclusion of the lemma.

Lemma 2.8 ([22], [23]). If $h \equiv 0$, then $f$ and $g$ share $1 C M$.

Lemma 2.9 ([18]). If $f, g$ share $1 C M$, then one of the following cases holds:

$$
\begin{aligned}
T(r, f)+T(r, g) \leq & 2\left\{N_{2}(r, 0 ; f)+N_{2}(r, 0 ; g)+N_{2}(r, \infty ; f)+N_{2}(r, \infty ; g)\right\} \\
& +S(r, f)+S(r, g)
\end{aligned}
$$

(ii) $f \equiv g$;

(iii) $f g \equiv 1$.

Lemma 2.10. If $\bar{E}_{4)}(1 ; f)=\bar{E}_{4)}(1 ; g)$ and $E_{2}(1 ; f)=E_{2)}(1 ; g)$ then the conclusion of Lemma 2.9 holds.

Proof. If $h \equiv 0$ then the result follows from Lemmas 2.8 and 2.9. So we suppose that $h \not \equiv 0$. Then by the second fundamental theorem and Lemmas 2.1 and 2.2 we get

$$
\begin{aligned}
T(r, f)+T(r, g) \leq & \bar{N}(r, 0 ; f)+\bar{N}(r, \infty ; f)+\bar{N}(r, 0 ; g)+\bar{N}(r, \infty ; g) \\
& +N(r, 1 ; f \mid=1)+\bar{N}(r, 1 ; f \mid \geq 2)+\bar{N}(r, 1 ; g) \\
& -N_{0}\left(r, 0 ; f^{\prime}\right)-N_{0}\left(r, 0 ; g^{\prime}\right)+S(r, f)+S(r, g)
\end{aligned}
$$




$$
\begin{aligned}
\leq & N_{2}(r, 0 ; f)+N_{2}(r, \infty ; f)+N_{2}(r, 0 ; g)+N_{2}(r, \infty ; g) \\
& +\bar{N}_{L}(r, 1 ; f)+\bar{N}_{L}(r, 1 ; g)+\bar{N}_{f \geq 5}(r, 1 ; f \mid g \neq 1) \\
& +\bar{N}_{g \geq 5}(r, 1 ; g \mid f \neq 1)+\bar{N}(r, 1 ; f \mid \geq 2)+\bar{N}(r, 1 ; g) \\
& +S(r, f)+S(r, g) .
\end{aligned}
$$

Since

$$
\begin{gathered}
\bar{N}(r, 1 ; f|=4 ; g|=3)+\bar{N}(r, 1 ; f \mid=4) \leq 2 \bar{N}(r, 1 ; f \mid=4), \\
\bar{N}(r, 1 ; f|=3 ; g|=4)+\bar{N}(r, 1 ; g \mid=4) \leq 2 \bar{N}(r, 1 ; g \mid=4),
\end{gathered}
$$

we see that

$$
\begin{aligned}
\bar{N}_{L}(r, 1 ; f)+\bar{N}_{L}(r, 1 ; g)+\bar{N} & f \geq 5 \\
& +\bar{N}(r, 1 ; f|g| \geq 2)+\bar{N}(r, 1 ; g) \\
\leq & \bar{N}(r, 1 ; f|=4 ; g|=3)+\bar{N}(r, 1 ; f \mid \geq 6)+\bar{N}(r, 1 ; g|=4 ; f|=3) \\
& +\bar{N}(r, 1 ; g \mid \geq 6)+\bar{N}(r, 1 ; f \mid \geq 5)+\bar{N}(r, 1 ; g \mid \geq 5)+\bar{N}(r, 1 ; f \mid=2) \\
& +\bar{N}(r, 1 ; f \mid=3)+\bar{N}(r, 1 ; f \mid=4)+\bar{N}(r, 1 ; f \mid \geq 5)+N(r, 1 ; g \mid=1) \\
& +\bar{N}(r, 1 ; g \mid=2)+\bar{N}(r, 1 ; g \mid=3)+\bar{N}(r, 1 ; g \mid=4)+\bar{N}(r, 1 ; g \mid \geq 5) \\
\leq & \frac{1}{2} N(r, 1 ; f \mid=1)+\frac{1}{2} N(r, 1 ; g \mid=1)+\bar{N}(r, 1 ; f \mid=2)+\bar{N}(r, 1 ; g \mid=2) \\
& +\bar{N}(r, 1 ; f \mid=3)+\bar{N}(r, 1 ; g \mid=3)+2 \bar{N}(r, 1 ; f \mid=4)+2 \bar{N}(r, 1 ; g \mid=4) \\
& +2 \bar{N}(r, 1 ; f \mid \geq 5)+2 \bar{N}(r, 1 ; g \mid \geq 5)+\bar{N}(r, 1 ; f \mid \geq 6)+\bar{N}(r, 1 ; g \mid \geq 6) \\
\leq & \frac{1}{2}[N(r, 1 ; f)+N(r, 1 ; g)] \leq \frac{1}{2}[T(r, f)+T(r, g)] .
\end{aligned}
$$

Now the lemma follows from (2.5).

Lemma 2.11 ([19]). Let $f$ be a nonconstant meromorphic function. Then

$$
N\left(r, 0 ; f^{(k)}\right) \leq k \bar{N}(r, \infty ; f)+N(r, 0 ; f)+S(r, f) .
$$

LEMMA $2.12([1])$. Let $f, g$ be two nonconstant meromorphic functions that share $(1,1)$ and have $h \not \equiv 0$. Then

$$
\begin{aligned}
T(r, f) \leq & N_{2}(r, 0 ; f)+N_{2}(r, \infty ; f)+N_{2}(r, 0 ; g)+N_{2}(r, \infty ; g) \\
& +\frac{1}{2} \bar{N}(r, 0 ; f)+\frac{1}{2} \bar{N}(r, \infty ; f)+S(r, f)+S(r, g) .
\end{aligned}
$$

LEMMA $2.13([1])$. Let $f, g$ be two nonconstant meromorphic functions that share $(1,0)$ and have $h \not \equiv 0$. Then

$$
\begin{aligned}
T(r, f) \leq & N_{2}(r, 0 ; f)+N_{2}(r, \infty ; f)+N_{2}(r, 0 ; g)+N_{2}(r, \infty ; g)+2 \bar{N}(r, 0 ; f) \\
& +2 \bar{N}(r, \infty ; f)+\bar{N}(r, 0 ; g)+\bar{N}(r, \infty ; g)+S(r, f)+S(r, g) .
\end{aligned}
$$


Lemma 2.14 ([1], cf. [21]). If $h \equiv 0$ and

$$
\limsup _{\substack{r \rightarrow \infty \\ r \in I}} \frac{\bar{N}(r, 0 ; f)+\bar{N}(r, \infty ; f)+\bar{N}(r, 0 ; g)+\bar{N}(r, \infty ; g)}{T(r)}<1,
$$

then $f \equiv g$ or $f g \equiv 1$.

LEMma 2.15 ([12]). Let $f$ and $g$ be two nonconstant meromorphic functions. Then

$$
f^{n}(f-1)^{2} f^{\prime} g^{n}(g-1)^{2} g^{\prime} \not \equiv 1
$$

for any integer $n \geq 7$.

Lemma 2.16 ([12]). Let

$F=f^{n+1}\left(\frac{f^{2}}{n+3}-\frac{2 f}{n+2}+\frac{1}{n+1}\right), \quad G=g^{n+1}\left(\frac{g^{2}}{n+3}-\frac{2 g}{n+2}+\frac{1}{n+1}\right)$, where $n \geq 5$ is an integer. Then $F^{\prime} \equiv G^{\prime}$ implies $F \equiv G$.

Lemma 2.17 ([12]). Let $F$ and $G$ be as in Lemma 2.16 and

$$
a=\frac{n+3}{n+2}+i \sqrt{\frac{n+3}{n+1}} \cdot \frac{1}{n+2} .
$$

Then

$$
\begin{aligned}
T(r, F) \leq & T\left(r, F^{\prime}\right)+N(r, 0 ; f)+N(r, a ; f)+N(r, \bar{a} ; f) \\
& -2 N(r, 1 ; f)-N\left(r, 0 ; f^{\prime}\right)+S(r, f) \\
T(r, G) \leq & T\left(r, G^{\prime}\right)+N(r, 0 ; g)+N(r, a ; g)+N(r, \bar{a} ; g) \\
& -2 N(r, 1 ; g)-N\left(r, 0 ; g^{\prime}\right)+S(r, g) .
\end{aligned}
$$

Lemma 2.18 ([12]). Let $F$ and $G$ be as in Lemma 2.16. Then $F \equiv G$ implies $f \equiv g$.

Lemma $2.19([8])$. Let $f, g$ share $(1,2)$. Then one of the following cases holds.

$$
\begin{aligned}
T(r) \leq & N_{2}(r, 0 ; f)+N_{2}(r, 0 ; g)+N_{2}(r, \infty ; f)+N_{2}(r, \infty ; g) \\
& +S(r, f)+S(r, g)
\end{aligned}
$$

(ii) $\quad f \equiv g$;

(iii) $f g \equiv 1$.

Lemma 2.20. Let $F$ and $G$ be as in Lemma 2.16. Then $S\left(r, F^{\prime}\right)=S(r, f)$ and $S\left(r, G^{\prime}\right)=S(r, g)$.

Proof. Using Lemma 2.4 we see that

$$
\begin{aligned}
& T\left(r, F^{\prime}\right) \leq 2 T(r, F)+S(r, F)=2(n+3) T(r, f)+S(r, f) \\
& T\left(r, G^{\prime}\right) \leq 2 T(r, G)+S(r, G)=2(n+3) T(r, g)+S(r, g) .
\end{aligned}
$$

Hence $S\left(r, F^{\prime}\right)=S(r, f)$ and $S\left(r, G^{\prime}\right)=S(r, g)$. 
Lemma 2.21 ([2]). Let $f$ and $g$ be two meromorphic functions, and $n \geq 8$ be an integer. Also let $F=f^{n}(f-1)^{2} f^{\prime}$ and $G=g^{n}(g-1)^{2} g^{\prime}$. If

$$
F=\frac{(b+1) G+(a-b-1)}{b G+(a-b)},
$$

where $a \neq 0, b$ are two constants, then $f \equiv g$.

\section{Proofs of the theorems}

Proof of Theorem 1.1. Let $F$ and $G$ be as in Lemma 2.16. Then it follows that $F^{\prime}$ and $G^{\prime}$ share $(1,2)$. So by Lemmas $2.11,2.17,2.19$ and 2.20 we obtain, for all $\varepsilon>0$,

$$
\begin{aligned}
T(r, F) \leq & T\left(r, F^{\prime}\right)+N(r, 0 ; f)+N(r, a ; f)+N(r, \bar{a} ; f) \\
& -2 N(r, 1 ; f)-N\left(r, 0 ; f^{\prime}\right)+S(r, f) \\
\leq & N_{2}\left(r, 0 ; F^{\prime}\right)+N_{2}\left(r, \infty ; F^{\prime}\right)+N_{2}\left(r, 0 ; G^{\prime}\right)+N_{2}\left(r, \infty ; G^{\prime}\right) \\
& +N(r, 0 ; f)+N(r, a ; f)+N(r, \bar{a} ; f)-2 N(r, 1 ; f) \\
& -N\left(r, 0 ; f^{\prime}\right)+S(r, f)+S(r, g) \\
\leq & 2 \bar{N}(r, 0 ; f)+2 \bar{N}(r, \infty ; f)+3 T(r, f)+2 \bar{N}(r, 0 ; g) \\
& +2 N(r, 1 ; g)+N\left(r, 0 ; g^{\prime}\right)+2 \bar{N}(r, \infty ; g) \\
& +S(r, f)+S(r, g) \\
\leq & 5 T(r, f)+5 T(r, g)+2 \bar{N}(r, \infty ; f)+3 \bar{N}(r, \infty ; g) \\
& +S(r, f)+S(r, g) \\
\leq & (7-2 \Theta(\infty ; f)+\varepsilon) T(r, f)+(8-3 \Theta(\infty ; g)+\varepsilon) T(r, g) \\
& +S(r, f)+S(r, g) \\
\leq & (15-2 \Theta(\infty ; f)-3 \Theta(\infty ; g)+2 \varepsilon) T(r)+S(r) .
\end{aligned}
$$

Using Lemma 2.4 we get

$$
(n+3) T(r, f) \leq(15-2 \Theta(\infty ; f)-3 \Theta(\infty ; g)+2 \varepsilon) T(r)+S(r) .
$$

In a similar manner we obtain

$$
(n+3) T(r, g) \leq\{15-3 \Theta(\infty ; f)-2 \Theta(\infty ; g)+2 \varepsilon\} T(r)+S(r) .
$$

Combining (3.2) and (3.3) we obtain

$$
\begin{aligned}
{[n-12+2 \Theta(\infty ; f)} & +2 \Theta(\infty ; g) \\
& +\min \{\Theta(\infty ; f), \Theta(\infty ; g)\}-2 \varepsilon] T(r) \leq S(r) .
\end{aligned}
$$

We choose

$$
0<\varepsilon<\frac{n-12+2 \Theta(\infty ; f)+2 \Theta(\infty ; g)+\min \{\Theta(\infty ; f), \Theta(\infty ; g)\}}{2} .
$$

Then (3.4) implies a contradiction and hence $H \equiv 0$. 
Therefore case (i) of Lemma 2.19 does not hold. According to Lemma $2.15, F^{\prime} \not \equiv G^{\prime}$. So case (iii) of Lemma 2.19 does not hold either. Hence we must have $F^{\prime} \equiv G^{\prime}$. Now the theorem follows from Lemmas 2.16 and 2.18.

Proof of Theorem 1.2. Let $F$ and $G$ be as in Lemma 2.16. Then $F^{\prime}$ and $G^{\prime}$ share $(1,1)$. Suppose $H \not \equiv 0$. Then by Lemma 2.12 we get

$$
\begin{aligned}
T\left(r, F^{\prime}\right) \leq & N_{2}\left(r, 0 ; F^{\prime}\right)+N_{2}\left(r, \infty ; F^{\prime}\right)+N_{2}\left(r, 0 ; G^{\prime}\right)+N_{2}\left(r, \infty ; G^{\prime}\right) \\
& +\frac{1}{2} \bar{N}\left(r, 0 ; F^{\prime}\right)+\frac{1}{2} \bar{N}\left(r, \infty ; F^{\prime}\right)+S\left(r, F^{\prime}\right)+S\left(r, G^{\prime}\right) .
\end{aligned}
$$

Now by Lemmas 2.4, 2.11, 2.17 and 2.20 and proceeding in the same way as for Theorem 1.1 we obtain, for all $\varepsilon>0$,

$$
(n+3) T(r, f) \leq[35 / 2-3 \Theta(\infty ; f)-3 \Theta(\infty ; g)+2 \varepsilon] T(r)+S(r) .
$$

In a similar manner we obtain

$$
(n+3) T(r, g) \leq[35 / 2-3 \Theta(\infty ; f)-3 \Theta(\infty ; g)+2 \varepsilon] T(r)+S(r) .
$$

From (3.5) and (3.6) we get

$$
[n-29 / 2+3 \Theta(\infty ; f)+3 \Theta(\infty ; g)-2 \varepsilon] T(r) \leq S(r) .
$$

Since $\varepsilon>0$ is arbitrary, (3.12) implies a contradiction. Hence $H \equiv 0$. Since

$$
\bar{N}\left(r, 0 ; f^{\prime}\right) \leq T\left(r, f^{\prime}\right)-m\left(r, 1 / f^{\prime}\right) \leq 2 T(r, f)-m\left(r, 1 / f^{\prime}\right)+S(r, f),
$$

we note that

$$
\begin{aligned}
\bar{N}(r, & \left.0 ; F^{\prime}\right)+\bar{N}\left(r, \infty ; F^{\prime}\right)+\bar{N}\left(r, 0 ; G^{\prime}\right)+\bar{N}\left(r, \infty ; G^{\prime}\right) \\
\leq & \bar{N}(r, 0 ; f)+\bar{N}(r, 1 ; f)+\bar{N}(r, \infty ; f)+\bar{N}\left(r, 0 ; f^{\prime}\right)+\bar{N}(r, 0 ; g) \\
& +\bar{N}(r, 1 ; g)+\bar{N}(r, \infty ; g)+\bar{N}\left(r, 0 ; g^{\prime}\right) \\
\leq & 5 T(r, f)+5 T(r, g)-m\left(r, 0 ; f^{\prime}\right)-m\left(r, 0 ; g^{\prime}\right)+S(r) \\
\leq & 10 T(r)-m\left(r, 0 ; f^{\prime}\right)-m\left(r, 0 ; g^{\prime}\right)+S(r) .
\end{aligned}
$$

Also using Lemma 2.4 we get

$$
\begin{aligned}
& T\left(r, F^{\prime}\right)+m\left(r, 1 / f^{\prime}\right) \\
& \quad=m\left(r, f^{n}(f-1)^{2} f^{\prime}\right)+m\left(r, 1 / f^{\prime}\right)+N\left(r, \infty ; f^{n}(f-1)^{2} f^{\prime}\right) \\
& \quad \geq m\left(r, f^{n}(f-1)^{2}\right)+N\left(r, \infty ; f^{n}(f-1)^{2}\right) \\
& \quad=T\left(r, f^{n}(f-1)^{2}\right)=(n+2) T(r, f)+O(1) .
\end{aligned}
$$

Similarly

$$
T\left(r, G^{\prime}\right)+m\left(r, 1 / g^{\prime}\right) \geq(n+2) T(r, g)+O(1) .
$$

From (3.9) and (3.10) we get

$$
\begin{aligned}
\max \left\{T\left(r, F^{\prime}\right)\right. & \left., T\left(r, G^{\prime}\right)\right\} \\
& \geq(n+2) T(r)-m\left(r, 1 / f^{\prime}\right)-m\left(r, 1 / g^{\prime}\right)+O(1) .
\end{aligned}
$$


By (3.8) and (3.11) applying Lemma 2.14 we get either $F^{\prime} \equiv G^{\prime}$ or $F^{\prime} G^{\prime} \equiv 1$. Now the theorem follows from Lemmas 2.15, 2.16 and 2.18.

Proof of Theorem 1.3. Let $F$ and $G$ be as in Lemma 2.16. Then $F^{\prime}$ and $G^{\prime}$ share $(1,0)$. Suppose $H \not \equiv 0$. Now using Lemma 2.13, follow the proof of Theorem 1.2.

Proof of Theorem 1.4. Let $F$ and $G$ be as in Lemma 2.16. From the assumptions it follows that $\bar{E}_{4)}\left(1 ; F^{\prime}\right)=\bar{E}_{4)}\left(1 ; G^{\prime}\right)$ and $E_{2)}\left(1 ; F^{\prime}\right)=E_{2)}\left(1 ; G^{\prime}\right)$. If possible suppose that

$$
\begin{aligned}
T\left(r, F^{\prime}\right) & +T\left(r, G^{\prime}\right) \\
\leq & 2\left\{N_{2}\left(r, 0 ; F^{\prime}\right)+N_{2}\left(r, 0 ; G^{\prime}\right)+N_{2}\left(r, \infty ; F^{\prime}\right)+N_{2}\left(r, \infty ; G^{\prime}\right)\right\} \\
& +S\left(r, F^{\prime}\right)+S\left(r, G^{\prime}\right) .
\end{aligned}
$$

Then by Lemmas 2.11, 2.17 and 2.20 we deduce from (3.12) that

$$
\begin{aligned}
T(r, F)+T(r, G) \leq & \left(r, F^{\prime}\right)+T\left(r, G^{\prime}\right)+N(r, 0 ; f)+N(r, a ; f) \\
& +N(r, \bar{a} ; f)-2 N(r, 1 ; f)-N\left(r, 0 ; f^{\prime}\right)+N(r, 0 ; g) \\
& +N(r, a ; g)+N(r, \bar{a} ; g)-2 N(r, 1 ; g)-N\left(r, 0 ; g^{\prime}\right) \\
& +S(r, f)+S(r, g) \\
\leq & 4 \bar{N}(r, 0 ; f)+4 N(r, 1 ; f)+2 N\left(r, 0 ; f^{\prime}\right)+4 \bar{N}(r, \infty ; f) \\
& +4 \bar{N}(r, 0 ; g)+4 N(r, 1 ; g)+2 N\left(r, 0 ; g^{\prime}\right)+4 \bar{N}(r, \infty ; g) \\
& +N(r, 0 ; f)+N(r, a ; f)+N(r, \bar{a} ; f)-2 N(r, 1 ; f) \\
& -N\left(r, 0 ; f^{\prime}\right)+N(r, 0 ; g)+N(r, a ; g)+N(r, \bar{a} ; g) \\
& -2 N(r, 1 ; g)-N\left(r, 0 ; g^{\prime}\right)+S(r, f)+S(r, g) \\
\leq & 10 T(r, f)+5 \bar{N}(r, \infty ; f)+10 T(r, g)+5 \bar{N}(r, \infty ; g) \\
& +S(r, f)+S(r, g) .
\end{aligned}
$$

So by Lemma 2.4 we get

$$
\begin{aligned}
(n-7) T(r, f)+ & (n-7) T(r, g) \\
\leq & 5 \bar{N}(r, \infty ; f)+5 \bar{N}(r, \infty ; g)+S(r, f)+S(r, g) .
\end{aligned}
$$

Choose $0<\varepsilon<n-12+\min \{\Theta(\infty ; f), \Theta(\infty ; g)\}$. Then from (3.13) we get

$$
\begin{array}{r}
(n-12+\Theta(\infty ; f)-\varepsilon) T(r, f)+(n-12+\Theta(\infty ; g)-\varepsilon) T(r, g) \\
\leq S(r, f)+S(r, g),
\end{array}
$$

which is a contradiction. Therefore the inequality (3.12) does not hold. So from Lemma 2.10 we see that either $F^{\prime} \equiv G^{\prime}$ or $F^{\prime} G^{\prime} \equiv 1$. Now the theorem follows from Lemmas 2.15, 2.16 and 2.18.

Proof of Theorem 1.5. Let $F$ and $G$ be as in Lemma 2.16. From the assumptions it follows that $\bar{E}_{3)}\left(1 ; F^{\prime}\right)=\bar{E}_{3)}\left(1 ; G^{\prime}\right)$ and $E_{1)}\left(1 ; F^{\prime}\right)=E_{1)}\left(1 ; G^{\prime}\right)$. 
Then by Lemmas 2.7, 2.17 and 2.20 we get

$$
\begin{aligned}
& T(r, F)+T(r, G) \\
& \leq 2\left\{N_{2}\left(r, 0 ; F^{\prime}\right)+N_{2}\left(r, 0 ; G^{\prime}\right)+N_{2}\left(r, \infty ; F^{\prime}\right)+N_{2}\left(r, \infty ; G^{\prime}\right)\right\} \\
& \quad+\frac{1}{2}\left[\bar{N}\left(r, 0 ; F^{\prime}\right)+\bar{N}\left(r, \infty ; F^{\prime}\right)+\bar{N}\left(r, 0 ; G^{\prime}\right)+\bar{N}\left(r, \infty ; G^{\prime}\right)\right] \\
& \quad+N(r, 0 ; f)+N(r, a ; f)+N(r, \bar{a} ; f)-2 N(r, 1 ; f)-N\left(r, 0 ; f^{\prime}\right) \\
& \quad+N(r, 0 ; g)+N(r, a ; g)+N(r, \bar{a} ; g)-2 N(r, 1 ; g)-N\left(r, 0 ; g^{\prime}\right) \\
& \quad+S(r, f)+S(r, g) .
\end{aligned}
$$

Using Lemmas 2.4 and 2.11 we infer from (3.14) that

$$
\begin{aligned}
(n+ & 3) T(r, f)+(n+3) T(r, g) \\
\leq & 4 \bar{N}(r, 0 ; f)+N(r, 0 ; f)+2 N(r, 1 ; f)+5 \bar{N}(r, \infty ; f)+3 T(r, f) \\
& +\frac{1}{2}[\bar{N}(r, 0 ; f)+\bar{N}(r, 1 ; f)+N(r, 0 ; f)+2 \bar{N}(r, \infty ; f)] \\
& +4 \bar{N}(r, 0 ; g)+N(r, 0 ; g)+2 N(r, 1 ; g)+5 \bar{N}(r, \infty ; g)+3 T(r, g) \\
& +\frac{1}{2}[\bar{N}(r, 0 ; g)+\bar{N}(r, 1 ; g)+N(r, 0 ; g)+2 \bar{N}(r, \infty ; g)]+S(r, f)+S(r, g) \\
\leq & \frac{23}{2} T(r, f)+\frac{23}{2} T(r, g)+6 \bar{N}(r, \infty ; f)+6 \bar{N}(r, \infty ; g)+S(r, f)+S(r, g),
\end{aligned}
$$
i.e.

$$
\begin{aligned}
& (n-17 / 2) T(r, f)+(n-17 / 2) T(r, g) \\
& \quad \leq 6 \bar{N}(r, \infty ; f)+6 \bar{N}(r, \infty ; g)+S(r, f)+S(r, g) .
\end{aligned}
$$

Choose $0<\varepsilon<n-29 / 2+6 \min \{\Theta(\infty ; f), \Theta(\infty ; g)\}$. Then from $(3.15)$ we get

$$
\begin{array}{r}
(n-29 / 2+6 \Theta(\infty ; f)-\varepsilon) T(r, f)+(n-29 / 2+6 \Theta(\infty ; g)-\varepsilon) T(r, g) \\
\leq S(r, f)+S(r, g),
\end{array}
$$

which is a contradiction. Therefore $H \equiv 0$. So

$$
F^{\prime}=\frac{(b+1) G^{\prime}+(a-b-1)}{b G^{\prime}+(a-b)},
$$

where $a \neq 0, b$ are two constants. Hence by Lemma 2.21 the theorem follows.

Acknowledgement. The author is grateful to Prof. M. L. Fang for supplying him the electronic file of the paper [2].

\section{References}

[1] A. Banerjee, Meromorphic functions sharing one value, Internat. J. Math. Math. Sci. 22 (2005), 3587-3598. 
[2] C. Y. Fang and M. L. Fang, Uniqueness of meromorphic functions and differential polynomials, Comput. Math. Appl. 44 (2002), 607-617.

[3] M. L. Fang and W. Hong, A unicity theorem for entire functions concerning differential polynomials, Indian J. Pure Appl. Math. 32 (2001), 1343-1348.

[4] W. K. Hayman, Meromorphic Functions, Clarendon Press, Oxford, 1964.

[5] I. Lahiri, Uniqueness of meromorphic functions when two linear differential polynomials share the same 1-points, Ann. Polon. Math. 71 (1999), 113-128.

[6] —, Value distribution of certain differential polynomials, Internat. J. Math. Math. Sci. 28 (2001), 83-91.

[7] -, Weighted sharing and uniqueness of meromorphic functions, Nagoya Math. J. 161 (2001), 193-206.

[8] -, Weighted value sharing and uniqueness of meromorphic functions, Complex Var. Theory Appl. 46 (2001), 241-253.

[9] —, Linear differential polynomials sharing the same 1-points with weight two, Ann. Polon. Math. 79 (2002), 157-170.

[10] I. Lahiri and A. Banerjee, Weighted sharing of two sets, Kyungpook Math. J. 46 (2006), 79-87.

[11] I. Lahiri and S. Dewan, Value distribution of the product of a meromorphic function and its derivative, Kodai Math. J. 26 (2003), 95-100.

[12] I. Lahiri and R. Pal, Nonlinear differential polynomials sharing 1-points, Bull. Korean Math. Soc. 43 (2006), 161-168.

[13] I. Lahiri and A. Sarkar, Nonlinear differential polynomials sharing 1-points with weight two, Chinese J. Contemp. Math. 25 (2004), 325-334.

[14] W. C. Lin, Uniqueness of differential polynomials and a problem of Lahiri, Pure Appl. Math. 17 (2001), 104-110 (in Chinese).

[15] W. C. Lin and H. X. Yi, Uniqueness theorems for meromorphic function, Indian J. Pure Appl. Math. 35 (2004), 121-132.

[16] A. Z. Mohon'ko, On the Nevanlinna characteristics of some meromorphic functions, Theory of Functions Funct. Anal. Appl. 14 (1971), 83-87.

[17] C. C. Yang, On deficiencies of differential polynomials II, Math. Z. 125 (1972), 107-112.

[18] C. C. Yang and X. H. Hua, Uniqueness and value sharing of meromorphic functions, Ann. Acad. Sci. Fenn. Math. 22 (1997), 395-406.

[19] H. X. Yi, Uniqueness of meromorphic functions and a question of C. C. Yang, Complex Var. Theory Appl. 14 (1990), 169-176.

[20] - On characteristic function of a meromorphic function and its derivative, Indian J. Math. 33 (1991), 119-133.

[21] - Meromorphic functions that share one or two values, Complex Var. Theory Appl. 28 (1995), 1-11.

[22] - Meromorphic functions that share one or two values II, Kodai Math. J. 22 (1999), 264-272.

[23] - Some further results on uniqueness of meromorphic functions, Complex Var. Theory Appl. 38 (1999), 375-385.

Department of Mathematics

Kalyani Government Engineering College

West Bengal 741235, India

E-mail: abanerjee_kal@yahoo.co.in, abanerjee@mail15.com 